\title{
Targeting postural reaction deficits in children with cerebral palsy: a case report
}

\author{
Meenakshi Batra ${ }^{1}$, Vijai Prakash Sharma ${ }^{2}$, Gyanendra Kumar Malik ${ }^{3}$, Vijay Batra ${ }^{4}$ \\ Sri Lanka Journal of Child Health, 2010; 40: 78-79
}

(Keywords: Postural reaction; cerebral palsy; postural control dynamics)

\begin{abstract}
This is a case report of a seven year old boy with cerebral palsy (CP) with a chief complaint of frequent falls and inability to walk independently without support. Gross motor milestones were delayed and on evaluation child demonstrated delayed, inappropriate and poorly developed postural reaction responses. To the best of knowledge of the authors, intervention in CP generally aims at managing tonal abnormalities, persistent pathological reflexes and achievement of motor milestones, but the key element for the development of motor control i.e. postural reaction deficits, is generally ignored. This case report emphasizes the need to assess postural reaction deficits and incorporate dynamics of postural control as an integral element for development / modification of postural control in CP.
\end{abstract}

\section{Introduction}

Postural reactions are the basic precursors of postural control. In children with $\mathrm{CP}$ postural reactions are either absent or poorly developed. Postural reactions automatically allow the body to adjust to the environment and maintain an upright equilibrium on which voluntary movements can be superimposed ${ }^{1,2}$. Postural reactions constitute a key element of postural control and include righting reaction, protective reaction \& equilibrium reactions. Hence intervention should aim at postural reactions besides other aspects of motor development.

${ }^{1}$ PhD Scholar, ${ }^{2}$ Director Professor, Department of Physical Medicine \& Rehabilitation, Rehabilitation \& Artificial Limb Centre, ${ }^{3}$ Director Professor \& Head Department of Paediatrics, ${ }^{4}$ Senior Research Fellow and PhD Scholar, Department of Physical Medicine \& Rehabilitation, Rehabilitation \& Artificial Limb Centre, Chhatrapati Shahuji Maharaj Medical University, India

(Received on 16 May 2010. Accepted on 24 June 2010)

\section{Case Report}

A seven year old boy with cerebral palsy (CP) with a chief parental complaint of frequent falls while walking came to the department for intervention. He had a full term normal delivery with delayed birth cry for which resuscitation was done. Gross motor developmental milestones were delayed. He achieved neck control at 7 months, rolling at 10 months, sitting at 36 months, quadruped at 40 months, crawling at 42 months, standing at 45 months and walking at 60 months of age. He had mild mental retardation; the intelligent quotient was 70 (as assessed by psychologist on VSMS). Muscle tone was assessed using Modified Ashworth Score (MAS) and child showed minimal increase in muscle tone in hip adductors, hamstrings and gastro-soleus (MAS grade1). The hip abductors and extensors, spinal extensors were weak (MRC-grade 2). The child used to sit in $\mathrm{W}$ - sitting position as a habitual posture.

Developmental reflexes were integrated and postural reactions assessed. Righting reactions were assessed by displacing the centre of gravity in lying and sitting positions on static support surface. He displayed partial inappropriate response with inability to restore normal body position on sudden displacement of centre of gravity. Protective reactions were assessed by applying sudden displacement stimuli in sitting and standing positions. In sitting position child displayed an inappropriate and delayed response, whereas in standing position he was unable to protect self from falling and demonstrated poor protective response. Equilibrium reactions were assessed on dynamic surface at varying angles with different intensities of stimuli (support surface displacement) in both sitting and standing positions. In sitting position he displayed a spontaneous inappropriate response by flexing the trunk with head and neck in hyperextension and holding the support surface by lowering the centre of gravity in order to prevent self from falling. In standing position he could only initiate but was not able to maintain centre of gravity.

Intervention began with an aim to improve postural reactions in order to achieve dynamic postural 
stability. The recruitment of axial muscles was done by adding end range resistance to both agonists and antagonists followed by sub-maximal resistance throughout the range. Simultaneously, upper and lower extremity supra-postural tasks along with postural muscle contraction were also added to recruit axial muscle. Later on bilateral resistance to both the limb girdles was added.

With improvement of postural stability the next goal was to facilitate multijoint interaction dynamics ${ }^{3,4}$. This was done by incorporating activities such as wall slide, wall pushing with knee partially flexed and ball throwing, alone or in combination. This was followed by enhancing the midrange resisted contraction to strengthen the muscles and promote co contraction surrounding a joint / girdle.

Finally, structural \& neuromuscular control was facilitated by adding vestibular, proprioceptive \& kinaesthetic input by varying stimulus characteristics (internal \& external), limb configuration and recruitment order with respect to support surface configuration, and perturbation in stable \& semistable pattern.

The intervention was given for two months. Reevaluation was done and child showed improved ability to produce: adequate weight shift, and adaptive postural responses, displaying significant qualitative improvement clinically. He was now able to display a normal appropriate response with an ability to right himself on sudden displacement of centre of gravity.

The protective reactions were also assessed. In sitting position he displayed normal appropriate response whereas in standing position he showed spontaneous but partially inappropriate response and was able to protect self from falling with minimal compensatory movements. The equilibrium reaction so observed in sitting position was found to be spontaneous and appropriate (with no postural fixation) on support surface displacement while in standing position child was now able to display spontaneous response with minimal compensation.

The postural reactions so developed had shown both quantitative and qualitative improvement resulting in better upright control and dynamic postural stability. The frequency of falling which was high (many times a day) initially had improved drastically and is now rarely in months on those similar set of activities.

\section{Discussion}

In CP, since movement variability and degree of freedom is severely affected ${ }^{2,5}$, the emphasis should be to work on postural reactions in order to achieve postural control and stability. Children with CP demonstrate alteration in timing of muscle activity in a functional synergy to produce movement ${ }^{3,4,6}$. Finally, limited degree of freedom decreases the number of strategies available for selection in CP. Constraints imposed by internal environment (e.g. musculoskeletal system, metabolic activity) and external environment (support surface, obstacles) limit the number of movement strategies. Hence dynamic activities which induce automatic postural adjustment at various positions and postures at different speeds and intensities should be practised. The combination of theses strategies produce the necessary variability in motor behaviour.

Intervention aiming at development / modification of postural reactions should be guided by models that include biomechanics, considerations of intersensory interaction and dynamic control mechanisms. Such an integrative conceptual framework will be helpful ${ }^{4,6}$. The importance of postural reaction should not be overlooked and there is a need to emphasize on the development / modification of postural reactions to improve postural dysfunction in children with $\mathrm{CP}$.

\section{References}

1. Haley MS. Sequential analysis of postural reactions in non-handicapped infants. Physiotherapy 1986: 66: $531-6$.

2. Mayston JM. People with CP. Effect of \& perspective for therapy. Neural Plast 2001: 8: 5169.

3. Horak FB, Nashner LM. Central programming of postural movements: Adaptation to altered support surface configurations. J Neurophysiol 1986; 55:1369-81.

4. Mergner T, Rosemeier T. Interaction of vestibular, somatosensory and visual signals for postural control and motion perception under terrestrial and microgravity conditions - a conceptual model. Brain Research Reviews 1998:28:118-35.

5. Longstaff A. Clinical notes of Neuroscience. 2nd ed. New York: Taylor\& Francis group; 2005.

6. Mijna Hadders-Algra. Development of postural control during the first18 months of life. Neural Plasticity 2005; 12 (2-3): 99-108. 